\title{
Plastronique 3D et 3D-MID, programme innovant d'enseignement supérieur et de formation à l'Université de Lyon
}

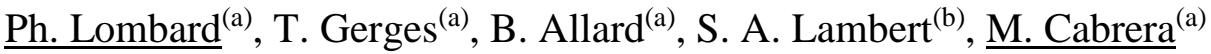

(a) Univ. Lyon, INSA Lyon, Ecole Centrale Lyon, Univ. Claude Bernard Lyon 1, CNRS, AMPERE.

F-69621 Villeurbanne, France

GIP-CNFM, Pôle CNFM de Lyon - CIMIRLY

Plateforme tech. scientifique \& pédagogique «Packaging avancé et Plastronique 3D »

(b) Univ Lyon, INSA Lyon, Univ Claude Bernard Lyon 1, CNRS, Univ Jean Monnet, INSERM, CREATIS, F-69616 Villeurbanne, France

Contacts : Responsables plateforme et référents pédagogique et scientifique
Philippe.lombard@univ-lyon1.fr Michel.cabrera@insa-lyon.fr

En 2015, une enquête menée par la Fondation pour l'Université de Lyon (1), auprès d'une centaine d'entreprises françaises, a montré que l'un des freins majeurs au développement de l'électronique sur substrat polymère, plus communément appelé plastronique, était le manque d'ingénieurs qualifiés ayant des compétences transdisciplinaires. Cela a été attribué à la nature intrinsèquement multi-physiques du sujet, impliquant de nombreux aspects qu'un ingénieur, non formé, peut difficilement gérer. En effet, la plastronique allie des compétences aussi bien en électronique, en plasturgie, en mécatronique, en sciences des matériaux, en chimie, etc. Une autre conclusion de cette enquête est que les entreprises ont besoin d'ingénieurs spécialisés dans tous ces domaines, mais également de responsables techniques capables de mener un projet en coopération avec les spécialistes concernés. Le défi consiste alors à proposer, en lien étroit avec les entreprises, des profils de collaborateurs appropriés capables de transcender le potentiel d'innovation de ces nouvelles technologies. Dans cet article, nous illustrerons un exemple de mini-projet réalisé sur la plateforme d'enseignement et de recherche dédiée à la plastronique sur LyonSaint-Etienne.

\section{Contexte et offre de formation}

L'Université de Lyon (IDEX Lyon Saint-Etienne) porte une formation spécialisée, non diplômante, portée par l'INSA Lyon pour le compte de l'INSA Lyon et l'école CPE. Ce projet s'appuie sur un ensemble de compétences et d'équipements localisés en Région Auvergne-Rhône-Alpes (AURA), à savoir la plateforme dédiée «packaging avancé et plastronique 3D»(située au laboratoire AMPERE - Campus LyonTech La doua, à Villeurbanne) ainsi que les moyens de plasturgie localisés à Oyonnax (INSA Lyon, le lycée Arbez Carme, la start-up S2P, le Centre Technique IPC et le laboratoire IMP). 
Cette action est financée par le Programme Investissement d'Avenir (PIA) français « The Plast to be » géré par Allize Plasturgie (principal syndicat du secteur de la plasturgie en France), le projet IDEFI-FINMINA, la région AURA, l'agglomération du Haut-Bugey (HB) ainsi qu'ACSIEL Alliance Electronique, l'UIMM de Lyon, Cap'tronic et le GIP CNFM.

\section{$\underline{\text { Introduction - Contexte et problématique }}$}

La plastronique ou 3D-MID (équivalent anglo-saxon du terme) (2-3) permet de doter de fonctions électroniques des objets en polymère (ou en composite) obtenus par différentes techniques de plasturgie, depuis la fabrication de pièces prototypes, notamment par impression 3D, jusqu'à la production de masse (thermo-injection, thermoformage, etc.). L'électronique permet de doter le système d'une " intelligence » tandis que la plasturgie assure les fonctions mécaniques et le packaging. Les interconnexions électriques peuvent être réalisées directement sur la surface du polymère par différents procédés (structuration laser, jet d'encres, plasma, dépôts divers, etc.). Il faut ensuite reporter et implémenter les composants (brasage, collage, clampage, etc.) avant d'effectuer les tests finaux (tests électriques, mécaniques, vieillissement, etc.).

La conception des systèmes est optimisée, la fonctionnalité est améliorée et de nouvelles fonctions peuvent être intégrés de manière optimales, et ce, tout en améliorant l'ergonomie et le design. L'assemblage est simplifié, la taille et le poids diminué. Cela permet une intégration optimale des fonctions hétérogènes (mécatroniques, optiques, fluidiques, etc.).

Des pièces plastroniques sont d'ores et déjà produites en grande série à l'instar des antennes électromagnétiques de tablettes et de smart phones. D'autres applications concernent les domaines de l'automobile et du médical. Des secteurs tels que : le transport, l'aéronautique et la défense, l'énergie, l'industrie et les biens d'équipements, l'électroménager et la domotique, le sport et les loisirs, etc., peuvent tirer profit de la plastronique pour déployer leur créativité.

La plastronique représente une véritable opportunité d'innovation pour l'industrie et le développement des plastiques intelligents, des objets connectés (tels que l'internet des objets (IoT)) et plus largement de l'électronique embarquée. Faut-il encore savoir faire les bons choix. En effet, produire de tels objets demande de faire travailler en synergie de nombreuses expertises techniques et cela dès la conception du produit. Le choix des technologies dépend de l'application visée, des fonctions à intégrer, du ou des matériaux, des dimensions et de la complexité des pièces et de l'électronique, ainsi que du volume et du coût de production à assurer.

Pour explorer pleinement le potentiel d'innovation de la plastronique, les entreprises doivent dès aujourd'hui pouvoir compter sur des collaborateurs avec des profils de compétences adaptés: connaissances techniques transversales, capacité à animer des projets dans un contexte interdisciplinaire, démarche d'innovation orientée produit, etc.

Les problématiques rencontrées dans la plastronique 3D sont en grande parties connexes à celles rencontrer dans les domaines tels que l'électronique sur films flexibles ou étirables, sur céramique ou encore sur textile. Nos compétences et champs d'actions sont donc élargis.

\section{L'offre de formation}

Illustrée sur la figure 1, l'offre de formation (4) est répartie sur deux semestres d'une année universitaire. Elle compte 372 heures de modules théoriques et pratiques pour un total de 54 crédits ECTS (European Credit Transfer and Accumulation System). 


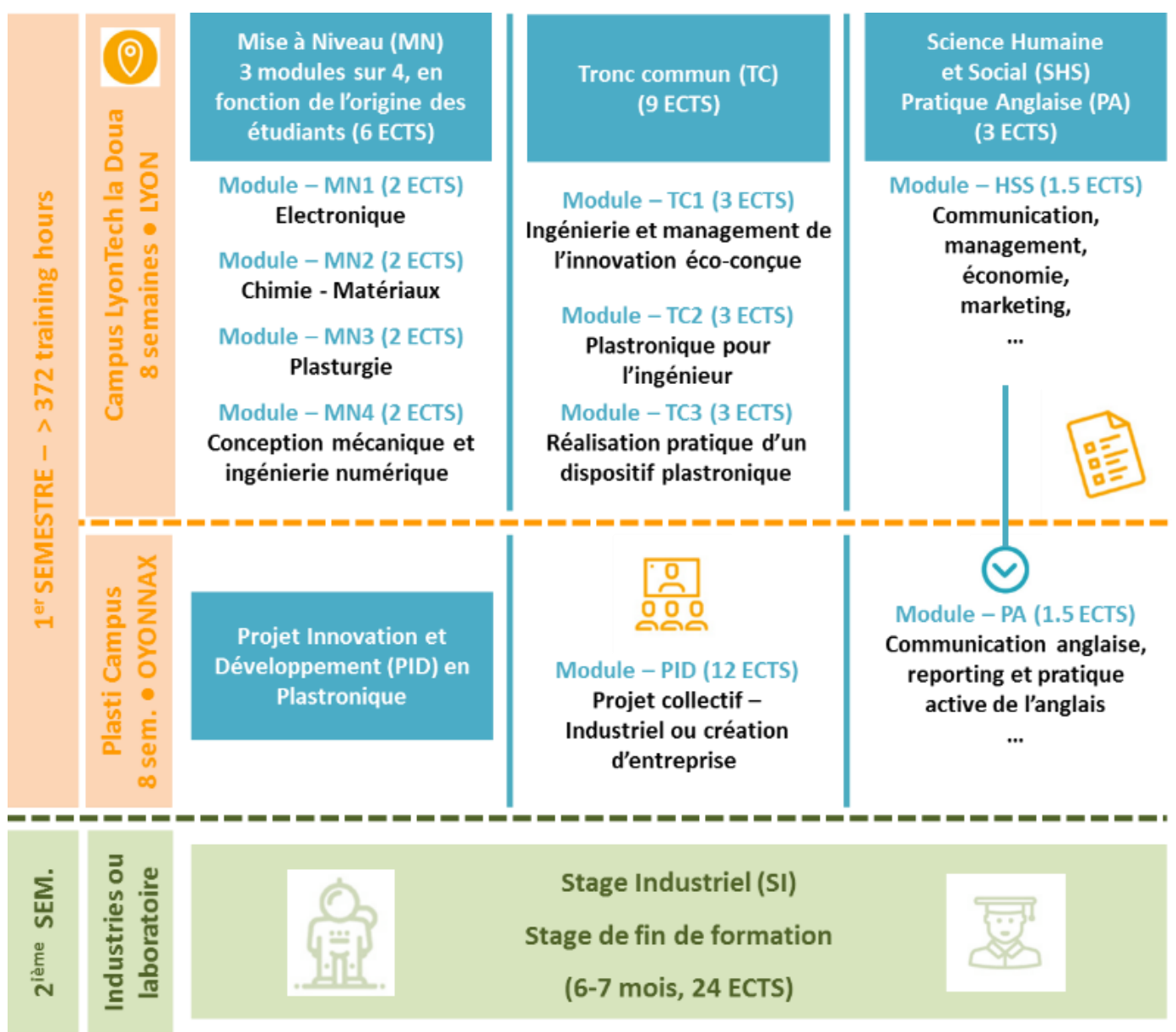

Fig.1. Déroulement du parcours « Chef de projet plastronique ».

Facile d'accès et ouvert à l'international, deux sites de formation sont concernés ; (i) Oyonnax, située dans la vallée du Haut-Bugey, est considérée comme la vallée française du plastique, (ii) Lyon (Campus Lyon-Tech la Doua, Villeurbanne), deuxième plus grande agglomération de France, est appréciée et reconnu pour sa formation scientifique et sa recherche en ingénierie. De plus ces deux sites jouissent d'une grande richesse industrielle dans de nombreux secteurs et activités économiques propices à l'émergence de la plastronique.

La première promotion (figure 2) a officiellement fait sa rentrée le 24 septembre 2018. Une première journée d'intégration, sur les sites de Lyon et d'Oyonnax, leur a permis de faire connaissance avec l'équipe pédagogique et de découvrir les principaux moyens techniques qu'ils seront amenés à utiliser au cours de leur formation. Conformément à nos attentes, cette promotion est composée de 11 élèves (6 étudiantes et 5 étudiants), de l'INSA et de l'école CPE de Lyon, présentant un large panel de compétences. La formation accueil ainsi des étudiants issus de domaine de formation variés : 2 en plasturgie, 1 en science et génie des matériaux, 1 en mécatronique, 1 en procédés et ingénierie, 5 en électronique et 1 en chimie.

Ravis d'avoir intégré cette première promotion, tous se sont montrés motivés par le caractère innovant des projets, le travail collaboratif, l'ouverture à d'autres disciplines et la proximité avec les entreprises. D'ici l'an prochaine, l'objectif est de former des promotions de 18 à 25 étudiants/an. 


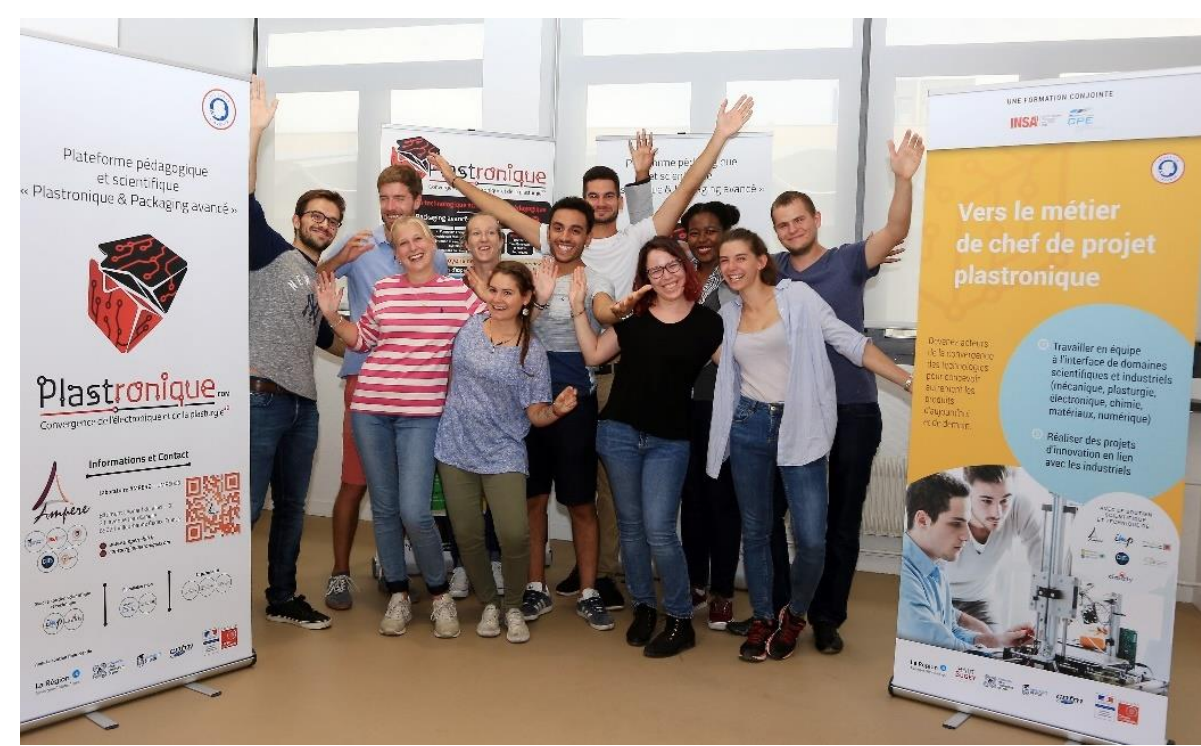

Fig.2. Première promotion lors de la journée d'intégration du 21 septembre 2018.

Les étudiants commencent à suivre des enseignements de remises à niveau sur des sujets extérieurs à leur cursus d'origine. Par exemple : une formation de base est dispensée en électronique, en mécanique et en traitement des matières plastiques pour les étudiants chimistes et ainsi de suite pour les autres étudiants (3 modules de remise à niveau sur 4). Cette première étape leur donne l'occasion d'étendre leurs compétences, de comprendre les concepts de bases, potentiels et verrous de chaque domaine scientifique. Cela leur permet également d'acquérir le vocabulaire clé, car la compréhension « de l'autre » est d'une importance fondamentale en plastronique.

Ensuite, ils suivent ensemble des cours approfondis sur la plastronique 3D. Nous abordons différentes techniques, processus, feuilles de route, etc., et discutons des forces et des faiblesses de chacun d'entre eux. Pour que les étudiants se familiarisent avec l'approche et la mise en œuvre de ces nouvelles technologies, un premier travail pratique concret (cf. l'exemple d'écrit ci-dessous) est réalisé étape par étape (de la conception aux tests électriques), en mélangeant plusieurs technologies (conventionnelles et plastroniques).

Des enseignements originaux comme la gestion de l'innovation et les aspects écologiques d'écoconception sont abordés. Des sciences humaines et sociales (gestion, marketing, etc.) et une connaissance active de l'anglais sont également proposées tout au long du $1^{\text {er }}$ semestre.

Des groupes de travail de 4 à 6 étudiants de différents horizons travaillent sur des projets innovants et problématiques proposés par des industriels en étroite collaboration avec des laboratoires de recherche tels qu'AMPERE (spécialisé dans l'électronique) et l'IMP (spécialisé dans la science des polymères). Tous les types de Projets d'Innovation et Développement (PID) sont les bienvenus; (i) du processus au système ; (ii) de l'étude exploratoire au prototype fonctionnel. En cohérence avec l'équipe encadrante, une approche pragmatique est appliquée; (i) de la technologie utilisée, à (ii) la fonction électronique considérée.

Enfin, les étudiants effectuent un stage durant tout le $2^{\text {ème }}$ semestre. Certains pourront être amenés à continuer leur mini-projet ou travailler sur un sujet de cœur (spécifique à leur cursus primaire). Ils peuvent également continuer en laboratoires sur un sujet plus fondamental.

La formation est ouverte aux étudiants en dernière année du cycle $\mathrm{M}$ que leur établissement diplômant envoie en «échange » pour deux semestres. Les étudiants sont 
donc diplômés de l'Université de Lyon avec un diplôme de leur formation d'origine (INSA ou CPE). La poursuite d'études en doctorat est envisageable.

Cette formation devrait être déclinée (licences, stages techniques, formations continues, etc.) et étendue (ouverture internationale) dans un proche avenir (horizon 2021). Dès aujourd'hui, nous sommes en mesure de nous adapter à un public large, dans le cadre d'une initiation ou d'un module pratique ciblé.

\section{Exemple de mini-projet projet d'étudiant}

\section{Contexte scientifique et enjeux}

En quatre décennies, l'Imagerie par Résonance Magnétique (IRM) est devenue un outil précieux et une référence dans de nombreux diagnostics médicaux. L'amélioration de la précision et l'acquisition quantitative des multiples paramètres dérivés de l'IRM ont été rendu possibles grâce aux très nombreux développements technologiques. Cependant, les performances des appareils d'IRM cliniques ne sont pas entièrement disponibles en IRM préclinique. Les principales raisons tiennent (i) à une taille plus réduite du marché et (ii) aux défis technologiques avec plus particulièrement la fabrication de dispositifs miniatures compacts, nécessaires à l'imagerie de petits échantillons. En conséquence, les instruments d'IRM précliniques sont en retard sur ceux cliniques. L'exemple traité ici a servi de démonstrateur pour le projet ANR JC ESTIMATE, en collaboration avec la plateforme « Packaging avancé et plastronique 3D », pour démontrer les avantages apportés par les technologies plastroniques et l'impression 3D pour la conception et la fabrication de sondes IRM multifonctionnelles. Ces technologies ont le potentiel d'améliorer et d'optimiser le processus de fabrication des sondes IRM pour une large gamme d'applications avec chacune ses contraintes spécifiques.

\section{Objectif et résultats}

L'objectif du mini-projet est de concevoir un système mécatronique complexe respectant les contraintes de l'environnement IRM.

Le système réalisé, illustré sur la figure 3 , doit notamment comprendre : des inductances $3 \mathrm{D}$ avec une capacité d'accord, un réseau fluidique instrumenté pour la régulation en température des échantillons imagés, un mouvement mécanique permettant d'ajuster le couplage inductif entre le coupleur et la bobine IRM, etc.

Après une étude mécanique, les pièces complexes du système sont fabriquées par impression 3D. Le réseau électronique et les bobines 3D sont réalisés et métallisés par des procédés plastroniques. Les composants électroniques sont reportés sur le système, sans adjonction de cartes électroniques. 


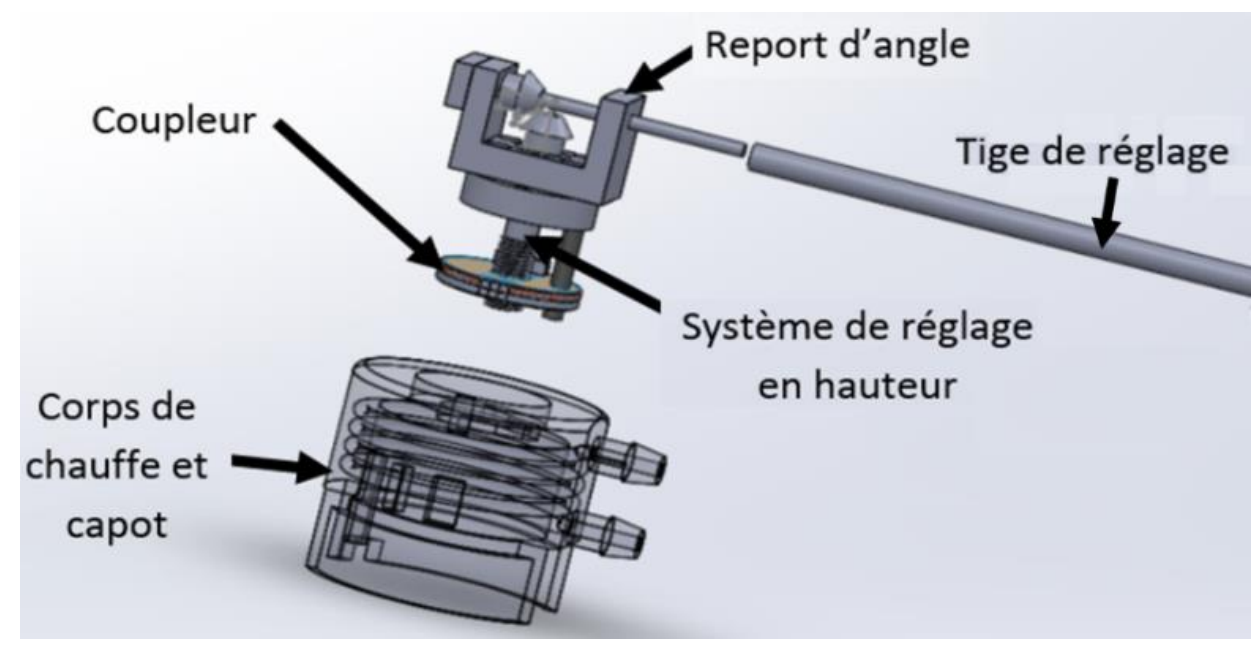

Fig.3. Vue CAO 3D du système complet. Le transfert d'angle permet de régler, à l'extérieur de l'IRM, l'inductance mutuelle (i.e. la distance) entre le coupleur, qui est positionné à l'intérieur du corps chauffant (canal fluidique), et la bobine IRM.

La figure 4 montre un prototype (réussi) avec des bobines plastronique 3D (bobine de Helmholtz) avec l'imagerie obtenue d'une cerise. Donc le système est fonctionnel.

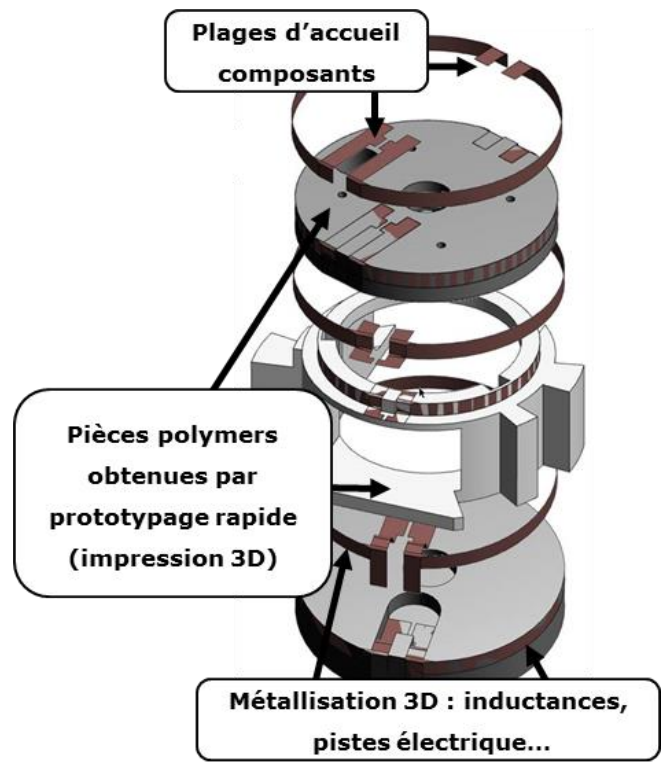

(a)

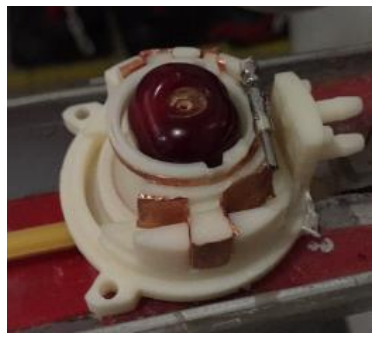

(b)

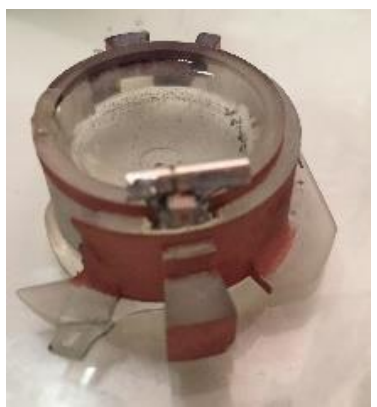

(c)

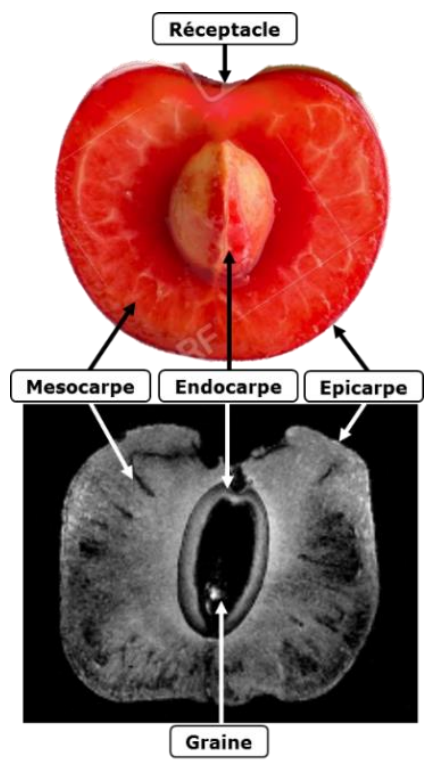

(d)

Fig.4. Dispositif plastronique 3D ; (a) vue CAO éclatée du coupleur avec pistes metallisées ; antennes IRM configurées avec des bobines 3D, composants, etc. (b) prototype «conventionnel », (c) prototype plastronique ; (d) imagerie ainsi obtenue, en son centre, d'une cerise (échantillon biologique), illustre la séquence d'imagerie complète. La taille de l'objet imagé est petite car le projet est axé sur l'imagerie de petits animaux ainsi que sur la recherche de tissus pour la médecine régénérative. (Séquence IRM réalisée par S.A. Lambert, porteur du projet ANR ESTIMATE).

Ces dispositifs ont été testés dans un IRM en conditions précliniques. Les premiers résultats obtenus sont très prometteurs et ont montré, a minima, des résultats aussi satisfaisants que ceux obtenus avec des méthodes conventionnelles (plus complexes à mettre en œuvre et moins reproductibles). 


\section{$\underline{\text { Mini-projet et modules de formation }}$}

Dans une problématique d'enjeux sociétaux, ce projet multi-physique et transdisciplinaire (mécanique, RF, fluidique, IRM, matériaux, métallisation, etc.) comporte de nombreux verrous scientifiques et technologiques.

Ces dispositifs ont été intégralement conçus et réalisés sur la plateforme «Packaging avancé et plastronique 3D» dans le cadre d'un mini-projet. Les résultats obtenus ont permis de montrer à des étudiants en formation la faisabilité de mener à bien un projet innovant dans son intégralité.

En termes de volume horaire et de quantité de travail ce mini-projet s'inscrit dans le module PID (Figure 1). De manière plus encadrée, un tel projet pourrait être décliné sous la forme de 4 séances de Travaux Pratiques (TP) et proposé dans un module plus court (une trentaine d'heures) à l'instar du module TC3 (Figure 1).

Le système présenté n'est évidemment qu'un exemple des multiples projets qu'il est possible de réaliser. En cohérence avec la complexité des systèmes et du volume horaire alloués, les étapes de réalisation peuvent être orientées en tenant compte de la pédagogie et domaines scientifiques à aborder, du niveau et de l'origine du publique accueilli.

La plateforme «packaging avancé et plastronique 3D » a pour vocation de s'ouvrir le plus largement possible en accueillant les partenaires académiques, scientifiques et industriels porteurs de projets.

\section{III.Moyens et ressources techniques unifiés}

Un ensemble de ressources du laboratoire à l'échelle industrielle est aujourd'hui mobilisé dans le cadre de la formation. Pour la partie plasturgie, les partenariats (plateformes dédiées polymères et composites, centres techniques, laboratoires, etc.) et les installations de fabrication et de caractérisation présentes à Oyonnax (Figure 5) sont utilisés pour la formation.

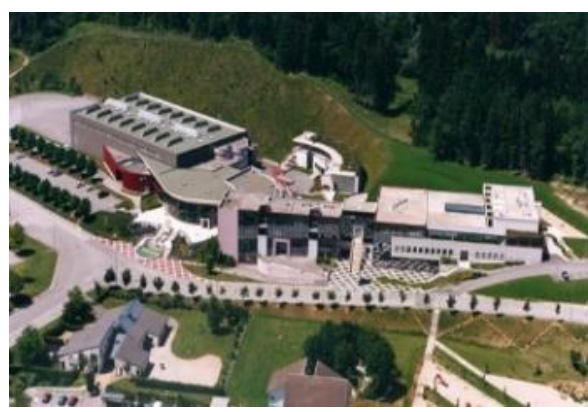

(a)

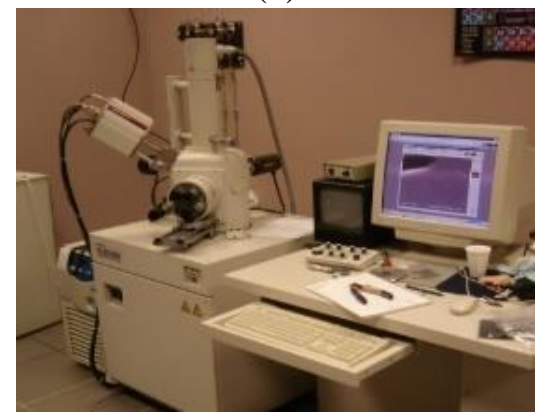

(c)

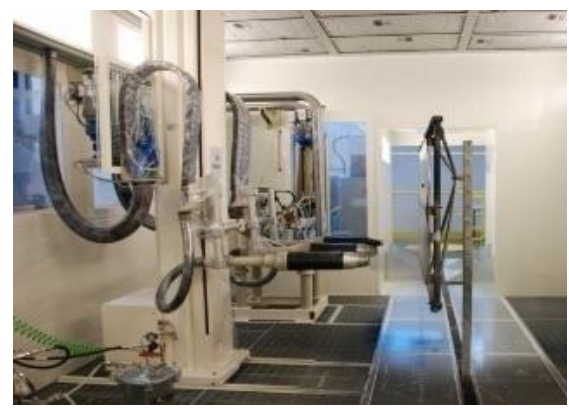

(b)

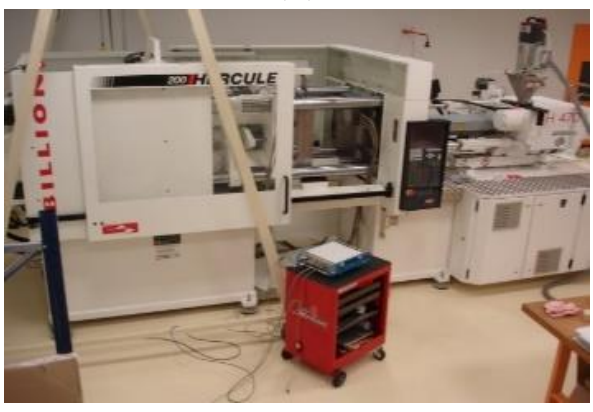

(d)

Fig.5. Site d'Oyonnax : (a) CT-IPC et l'antenne de l'INSA ; (b) Platforme «Coating Expert »; (c) Ex. de ressources au laboratoire IMP (ex. MEB) et (d) à l'INSA (presse à injecter, extrudeur, RTM, etc.). 
La plupart des investissements sont néanmoins réalisés à Lyon, via la mise en place d'une plate-forme pédagogique baptisée «Packaging avancé et plastronique 3D » illustrée sur la figure 6. Cela permet de regrouper dans un même lieu tous les outils et compétences nécessaires en électronique pour développer des dispositifs et des systèmes, de la conception aux tests et à la caractérisation.

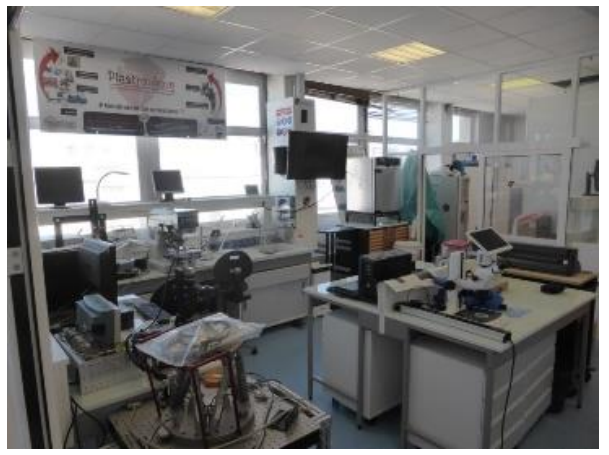

(a)

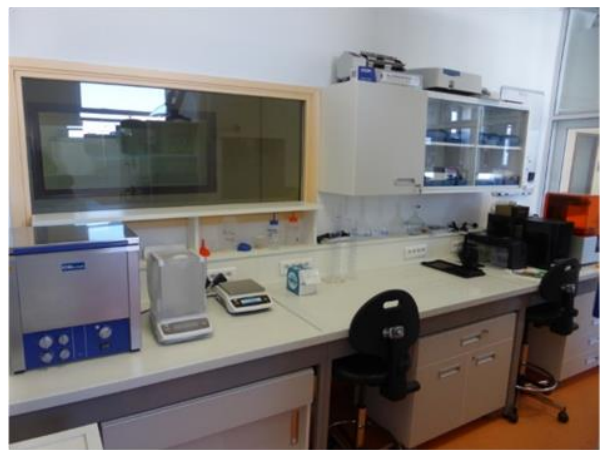

(c)

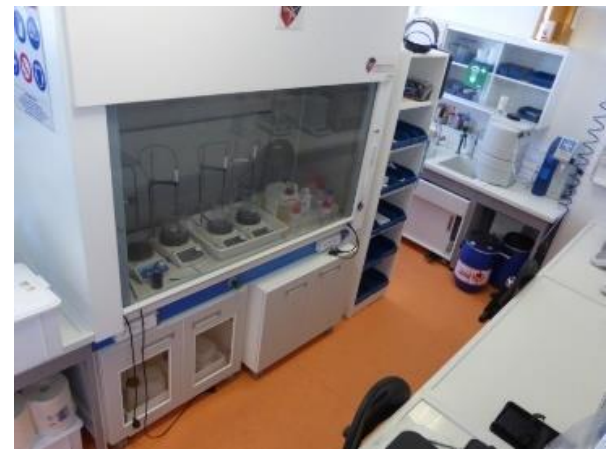

(b)

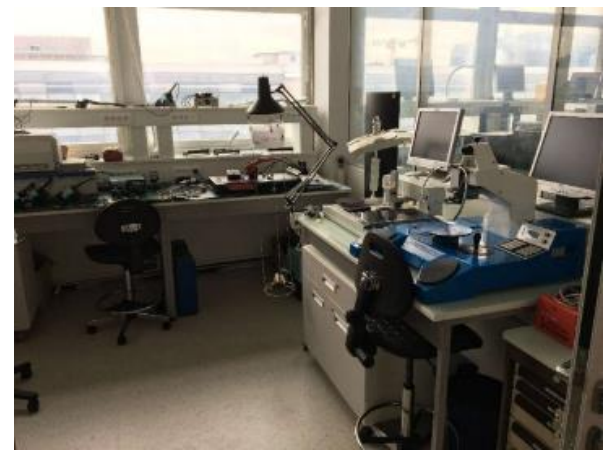

(d)

Fig.6. Plateforme «Packaging avancé et plastronique 3D » (lab. AMPERE) : (a) pôle de «fabrication et de structuration $3 \mathrm{D}$ », (b \& c) pôle de chimie (traitement de surface, métallisation chimique, électrodéposition, etc.), (d) pôle «implémentation et intégration » (mise en œuvre, test et caractérisation).

D'une surface supérieure à $100 \mathrm{~m}^{2}$, cette plateforme est divisée en quatre pôles de travail :

- un pôle «fabrication et structuration $3 \mathrm{D}$ »; fabrication de pièces et structuration du réseau conducteur, etc. ;

- un pôle chimie ; traitement de surface, métallisation chimique, électrodéposition, etc. ;

- un pôle « implémentation et intégration » comprenant, entre autres, le dépôt et la mise en œuvre de composants, la brasure et la mise en œuvre de fonctions complexes ;

- un laboratoire dédié à la caractérisation électrique et mécanique ainsi qu'à l'inspection des systèmes. A terme, les aspects d'étude en vieillissement seront également intégrés.

Non-exhaustive, la figure 7 présente une liste des moyens acquis et en cours d'acquisition de la plateforme « Packaging avancé et plastronique 3D ». Ces moyens sont classés par grands axes : - fabrication et structuration 3D - chimie - électronique caractérisation et inspection.

Une multitude d'autres instruments et appareils présents au laboratoire AMPERE (ex. MEB, chambres d'essais radiofréquence (RF) et haute tension (HT), etc.) sont accessibles aux apprenants lors de la formation et des projets industriels. 


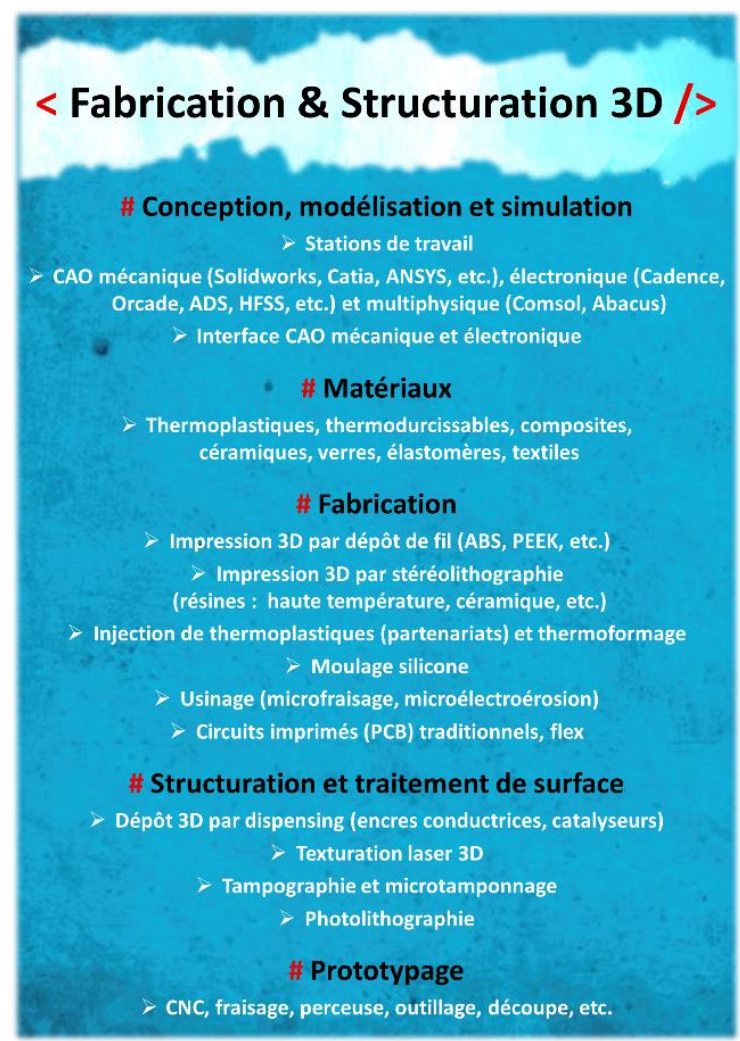

(a)

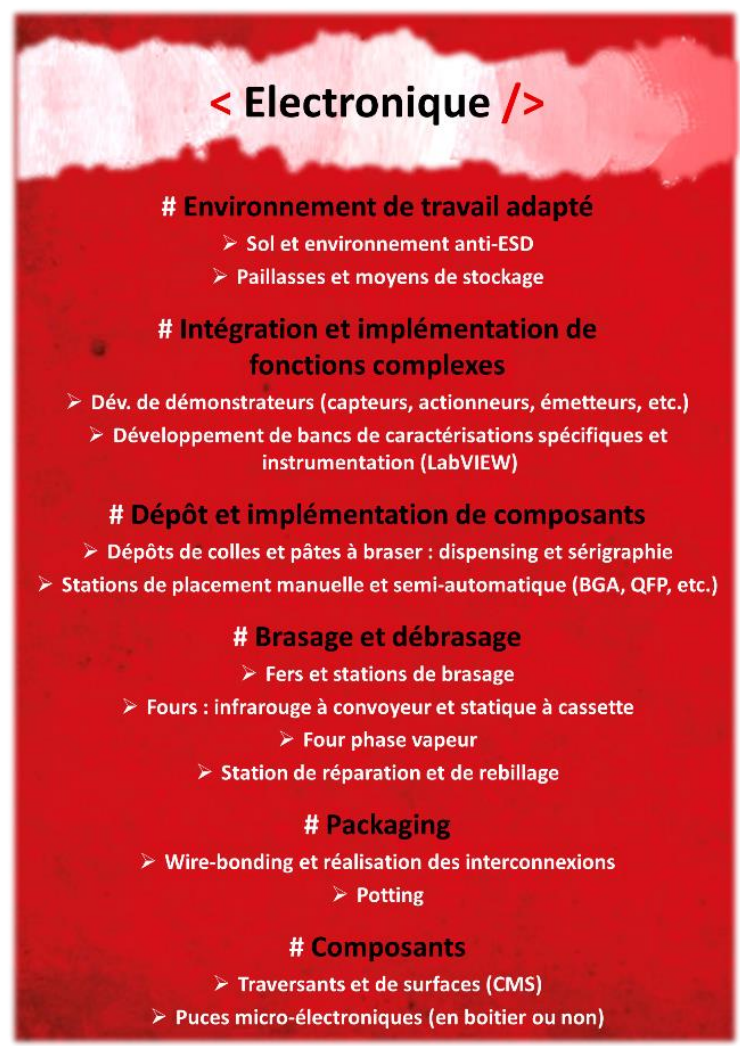

(c)

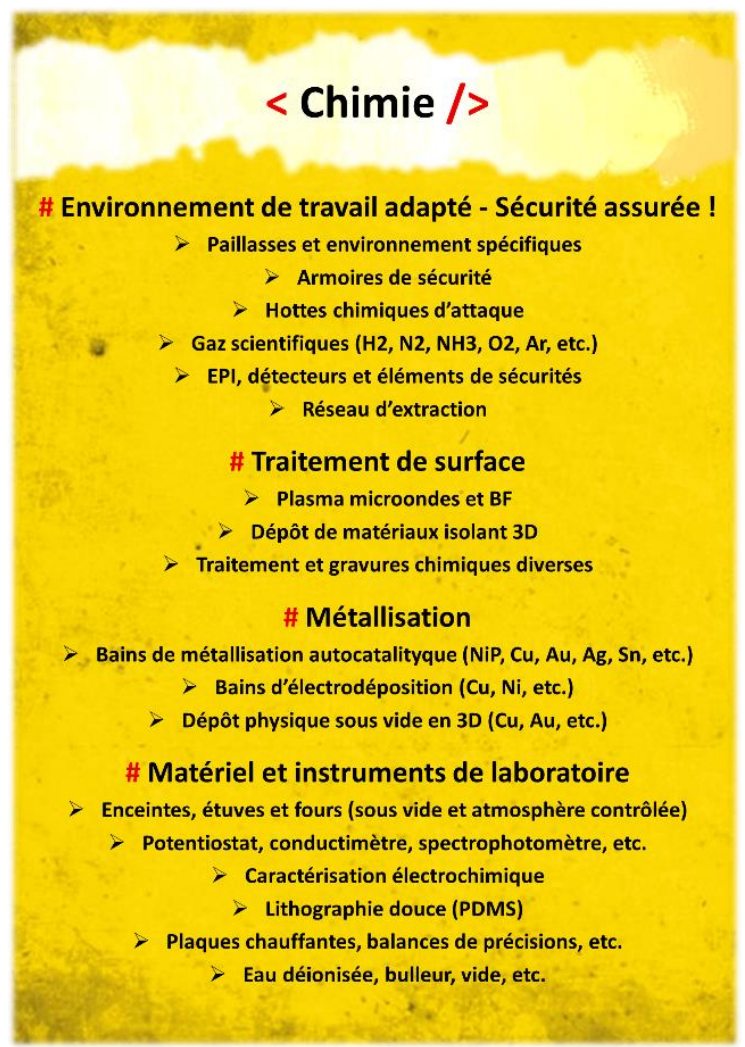

(b)

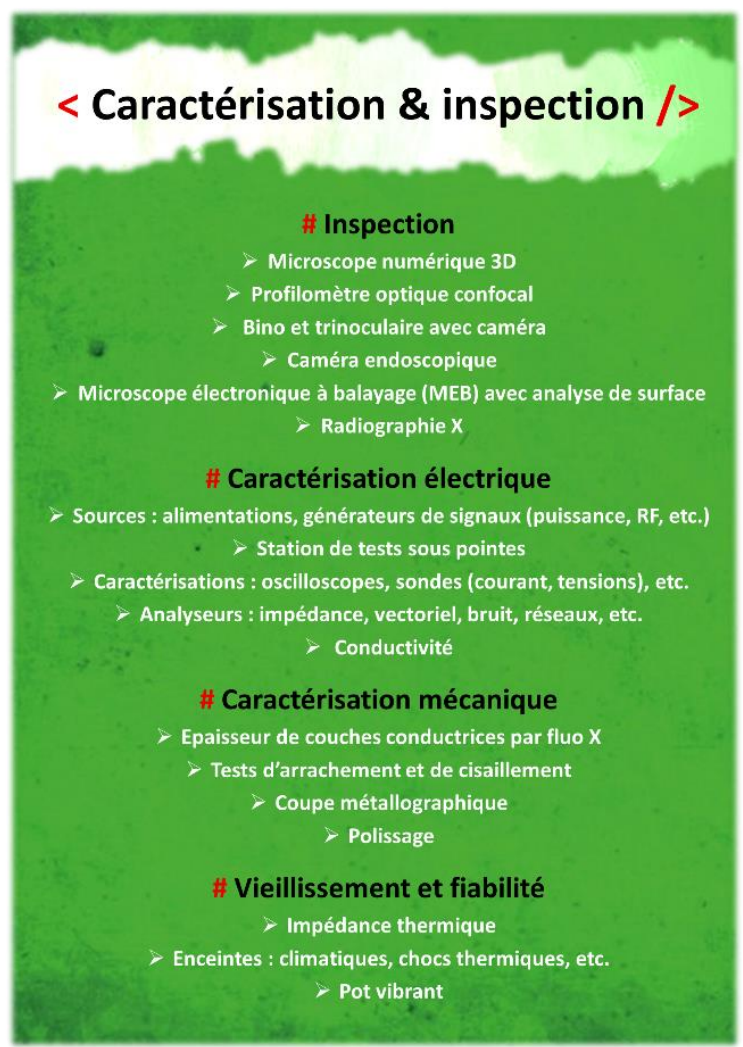

(d)

Fig.7. Liste, non-exhaustive, des moyens acquis et en cours d'acquisition de la plateforme «Packaging avancé et plastronique 3D» (lab. AMPERE) : (a) fabrication et structuration 3D; (b) chimie ; (c) électronique ; (d) caractérisation et inspection. 


\section{IV.Conclusion}

Les technologies plastroniques modifient profondément la façon de concevoir les systèmes. Les plastiques deviennent plus «intelligents » et les systèmes électroniques peuvent être mis en œuvre sur des objets en polymères de formes 3D complexes. Dans ce sens, l'innovation ne fait que commencer!

Un programme de formation ambitieux a été présenté. Il a été développé par l'Université de Lyon en collaboration avec de nombreux partenaires (industries, laboratoires, fondation, etc.). Après une phase de mise à niveau, les étudiants suivent une formation spécifique leur permettant d'élargir leurs compétences pour pouvoir gérer un projet complet. D'autre part, des groupes de travail d'étudiants travaillent sur des projets industriels proposés par des entreprises. Une approche pragmatique et de nombreux moyens, dont une plateforme dédiée, «Packaging avancé et plastronique 3D », sont mis à leur disposition. Les étudiants pourront ainsi devenir chefs de projets plastronique.

\section{Remerciements}

Les auteurs remercient tout particulièrement le Ministère français de l'Enseignement Supérieur, de la Recherche et de l'Innovation, le PIA et tous les membres du comité de pilotage du projet «The Plast to Be », en particulier: A. Merle, de la Fondation pour l'Université de Lyon ; J-Y Charmeau, de l'INSA ; N. Abouchi, de CPE ; ainsi que V. Semet et R. Delamea pour leur contribution au projet IRM.

Les auteurs sont également reconnaissants au GIP-CNFM et au programme IDEFIFINMINA (6), à la région Auvergne Rhône-Alpes et à celle du Haut-Bugey, à l'Université de Lyon, mais également à l'INSA de Lyon et l'école CPE, le lycée Arbez Carme, tous les laboratoires, plates-formes et centres techniques partenaires (AMPERE, IMP, CNRS, CIMIRLY, CT-IPC, etc.), ainsi que les représentants de branches et organismes de valorisation (Plastipolis, Fédération de la plasturgie et des composites, Allizé Plasturgie, ACSIEL, UIMM, Cap'tronic, etc.).

Enfin, ils remercient vivement tous les sponsors et partenaires industriels engagés à leurs côtés dans ce projet, sans qui ce travail et leurs efforts n'auraient aucun sens. Il faut citer en particulier : Plastic Omnium, le groupe Seb, la fondation Dassault Systems, SintexNP, Cirly, S2P, sans oublier ceux qui sont en cours d'adhésion au projet.

Pour toutes ces raisons et pour leur soutien au quotidien : un grand merci !

\section{Références}

1. Website - http://www.fondation-pour-universite-lyon.org.

2. Ph. Lombard, M. Cabrera, V. Semet, B. Allard, S. Kamotesov, M. Moguedet and J-Y Charmeau, "Plateforme pédagogique et scientifique; Exemple de conception, réalisation et caractérisation d'un capteur inductif multidirectionnel de proximité pour la détection d'approche 3D," J3eA, vol 16, 2017, JPCNFM $2016-14 \mathrm{e}$ journées pédagogiques du CNFM - Website https://doi.org/10.1051/j3ea/20171013, (2017).

3. Ph. Lombard, V. Semet, M. Cabrera, "Formation au prototypage de systèmes électroniques $2 D$ et 3D. Initiation à la Plastronique 3 D et aux dispositifs MID ”, J3eA, vol 14, 2015, JPCNFM 2014 - 13 journées pédagogiques CNFM - Website - https://doi.org/10.1051/j3ea/2015025, (2015).

4. Website - https://www.universite-lyon.fr/formation/formations-innovantes/plastronique-3d10618.kjsp?RH=1486387482944.

5. Chaîne vidéo, www.youtube.com/channel/UCYEgiR9FiVF3Wqc64ie7W6g/videos.

6. ANR IDEFI-FINMINA : Initiative d'Excellence - Formation Innovante en MIcroélectronique et Nanotech., ANR-11-IDFI-0017. http://www.cnfm.fr/VersionFrancaise/actualites/FINMINA.htm. 